\title{
QUANTITATIVE DETERMINATION OF AMLODIPINE FROM CACO-2 CELL MONOLAYERS BY HIGH-PERFORMANCE LIQUID CHROMATOGRAPHY-MASS SPECTROMETRY/MASS SPECTROMETRY
}

\author{
LILIYA LOGOYDA* \\ Department of Pharmaceutical Chemistry, I. Horbachevsky Ternopil State Medical University, Ternopil, Ukraine.
} Email: logojda@tdmu.edu.ua

Received: 29 January 2018, Revised and Accepted: 23 February 2018

\section{ABSTRACT}

Objective: A simple, rapid high-performance liquid chromatography-mass spectrometry/mass spectrometry (MS/MS) method was developed for the determination of amlodipine from confluent Caco-2 monolayers and from aqueous solution.

Methods: Chromatography was achieved on Discovery C18, 50×2.1 mm, $5 \mu \mathrm{m}$ column. Samples were chromatographed in a gradient mode (eluent A [acetonitrile-water-formic acid, 5:95:0.1 v/v], eluent B [acetonitrile-formic acid, 100:0.1 v/v]). The initial content of the eluent B is $0 \%$, which increases linearly by $1.0 \mathrm{~min}$ to $100 \%$ and to $1.01 \mathrm{~min}$ returns to the initial $0 \%$. The mobile phase was delivered at a flow rate of $0.400 \mathrm{ml} / \mathrm{min}$ into the MS electrospray ionization chamber. The sample volume was $5 \mu$ l.

Results: Under these conditions, amlodipine was eluted at $1.61 \mathrm{~min}$. According to the Caco-2 test results, amlodipine appeared to have moderate to high permeability. Recovery value for amlodipine is $52.33 \%$. Caco-2 permeability values for amlodipine are in agreement with BCS Class I classification for these drugs and their known high bioavailability in humans.

Conclusion: A new rapid method was developed for the determination of amlodipine from confluent Caco-2 monolayers and from aqueous solution. Acquired results demonstrate that proposed strategy can be effortlessly and advantageously applied for the examination of amlodipine from Caco-2 cell monolayers.

Keywords: Amlodipine besylate, High-performance liquid chromatography-mass spectrometry/mass spectrometry, Caco-2 cells, Bioavailability.

(c) 2018 The Authors. Published by Innovare Academic Sciences Pvt Ltd. This is an open access article under the CC BY license (http://creativecommons. org/licenses/by/4. 0/) DOI: http://dx.doi.org/10.22159/ajpcr.2018.v11i6.24971

\section{INTRODUCTION}

Combination of the in vitro models that are high throughput but less predictive and the in vivo models that are low throughput but more predictive is used effectively to evaluate the intestinal permeability and transport characteristics of a large number of drug candidates during lead selection and lead optimization processes. Parallel artificial membrane permeability assay and Caco- 2 cells are the most frequently used in vitro models to assess intestinal permeability. The popularity of these models stems from their potential for high throughput, costeffectiveness, and adequate predictability of absorption potential in humans. However, several caveats associated with these models (e.g., poor predictability for transporter-mediated and paracellularly absorbed compounds, significant non-specific binding to cells/devices leading to poor recovery, and variability associated with experimental factors) need to be considered carefully to realize their full potential. P-glycoprotein, among other pharmaceutically relevant transporters, has been well demonstrated to be the major determinant of drug disposition [1].

There is an high-performance liquid chromatography (HPLC) method described for the determination of amlodipine (Fig. 1) in pharmaceutical preparations. In addition, there are another methods reported for the determination of amlodipine in human plasma [1-3].

However, both methods are not developed for the examination of amlodipine from Caco- 2 cell monolayers. Therefore, the aim of this study was to develop and validate an efficient HPLC-mass spectrometry/mass spectrometry (MS/MS) method for the determination of amlodipine from Caco-2 cell monolayers.

\section{METHODS}

\section{Reagents and consumables}

Trypsin EDTA (10x) 0.5\%/0.2\% in DPBS (PAA, UK; Cat\# L11-003)

HEPES, high purity grade (Helicon, Am-0485)

Dulbecco's PBS (1x) without Ca and Mg (PAA, UK; Cat\# H15-002)

Hanks' BSS (1x) without Ca and Mg without phenol red (PAA, UK; Cat\# H15-009)

DMSO Chromasolv Plus, HPLC grade, $\geq 99.7 \%$ (Sigma-Aldrich, USA; Cat \#34869)

DMEM (4.5g/l) liquid without L-Glutamine (PAA, UK; Cat\# E15-009)

L-Glutamine (200 mM) (PAA, UK; Cat\# M11-004)

Fetal Bovine Serum “GOLD” EU approved (PAA, UK; Cat\# A15-151)

Penicillin/Streptomycin (100x) (PAA, UK; Cat\# P11-010)

Acetonitrile Chromasolv, gradient grade, for HPLC, $\geq 99.9 \%$ (SigmaAldrich, USA; Cat \#34851)

Formic acid for MS, 98\% (Fluka, USA; Cat \#94318)

Falcon ${ }^{\circledR}$ HTS 24-multiwell insert systems with media feeder tray (BD Biosciences, USA; Prod\# 351181)

Falcon ${ }^{\circledR} 24$-Well TC-Treated Cell PS Permeable Support Companion Plate (BD, Prod\# 353504)

Centrifuge tubes, $50 \mathrm{ml}$ (Santa Cruz, USA; Cat\# sc-200251)

Serological pipettes $5 \mathrm{ml}, 10 \mathrm{ml}$, and $25 \mathrm{ml}$ (Greiner Bio-One)

Disposable pipettor tips (Thermo Scientific, Fisherbrand, Eppendorf, USA)

$1.1 \mathrm{ml}$ microtubes in microracks (Thermo Scientific, USA)

Zorbax Eclipse Plus C18 column 2.1×50 mm, $3.5 \mu$ m (Agilent Technologies, Inc., USA) 
Propranolol hydrochloride $\geq 99 \%$ (TLC), powder (Sigma-Aldrich, USA; Cat \# P0884)

Quinidine anhydrous (Sigma-Aldrich, USA; Cat \# Q3625 Lot \#BCBF1345V) Atenolol, analytical reference material, $\geq 98.5 \%$ (HPLC) (Sigma-Aldrich, USA; Cat \#74827)

Test compound was provided as dry powder (salt form amlodipine besylate) and was dissolved in DMSO at $10 \mathrm{mM}$ to prepare working stocks.

\section{Analytical system}

All measurements were performed using Shimadzu VP HPLC system including vacuum degasser, gradient pumps, reverse phase HPLC column, column oven, and autosampler. The HPLC system was coupled with tandem MS API 3000 (PE Sciex). The TurboIonSpray ion source was used in both positive and negative ion modes. Parameters of electrospray ionizer (ESI) and multiple reaction monitoring (MRM) are listed in Tables 1 and 2. Acquisition and analysis of the data were performed using analyst 1.5 .2 software (PE Sciex). Chromatography was achieved on Discovery C18, 50×2.1 mm, $5 \mu \mathrm{m}$ column. Samples were chromatographed in a gradient mode (eluent A [acetonitrile-water-formic acid, 5:95:0.1 v/v], eluent B [acetonitrile-formic acid, 100:0.1 v/v]). The initial content of the eluent B is $0 \%$, which increases linearly by $1.0 \mathrm{~min}$ to $100 \%$ and to $1.01 \mathrm{~min}$ returns to the initial $0 \%$. The mobile phase was delivered at a flow rate of $0.400 \mathrm{ml} / \mathrm{min}$ into the MS ESI chamber. The sample volume was $5 \mu \mathrm{l}$

Caco- 2 cells were cultivated in $75 \mathrm{~cm}^{2}$ flasks to $70-80 \%$ of confluence according to the ATCC and Millipore recommendations [2] in humidified atmosphere at $37^{\circ} \mathrm{C}$ and $5 \% \mathrm{CO}_{2}$. Cells were detached with trypsin/EDTA solution and resuspended in the cell culture medium to a final concentration of $2 \times 10^{5}$ cells $/ \mathrm{ml} .500 \mu \mathrm{l}$ of the cell suspension was added to each well of HTS 24-multiwell insert system and $35 \mathrm{ml}$ of prewarmed complete medium was added to the feeder tray. Caco2 cells were incubated in multiwell insert system for 21 days before the transport experiments. The medium in filter plate and feeder tray was changed every other day. After 21 days of cell growth, the integrity of the monolayer was verified by measuring the transepithelial electrical resistance (TEER) for every well using the Millicell-ERS system ohm meter. The final TEER values were within the range of 150-600 $\Omega \times \mathrm{Cm}^{2}$ [3] as required for the assay conditions. 24-well insert plate was removed from its feeder plate and placed in a new sterile 24-well transport analysis plate. The medium was aspirated and inserts washed with PBS twice.

To determine the rate of compounds transport in apical (A) to basolateral (B) direction, $300 \mu \mathrm{L}$ of the test compound dissolved in transport buffer at $10 \mu \mathrm{M}$ (HBSS, $10 \mathrm{mM}$ HEPES, $\mathrm{pH}=7.4$ ) was added into the filter wells; $1000 \mu \mathrm{L}$ of buffer (HBSS, $10 \mathrm{mM}$ HEPES, pH=7.4) was added to transport analysis plate wells. The plates were incubated for $90 \mathrm{~min}$ at $37^{\circ} \mathrm{C}$ with shaking at $100 \mathrm{rpm} .75 \mu \mathrm{L}$ aliquots were taken from the donor and receiver compartments for LC-MS/MS analysis. All samples were mixed with two volumes of acetonitrile with following protein sedimentation by centrifuging at $10,000 \mathrm{rpm}$ for $10 \mathrm{~min}$. Supernatants were analyzed using the HPLC system coupled with tandem MS.

Propranolol (high permeability), atenolol (low permeability), and quinidine (moderate permeability) were used as reference compounds.

The apparent permeability $\left(\mathrm{P}_{\text {app }}\right)$ was calculated for Caco-2 permeability assay using the following equation:

\section{$P_{\text {app }}=\frac{V_{A}}{\text { Area } \times \text { time }} \times \frac{[\mathrm{drug}]_{\text {acc }}}{[\text { drug }]_{\text {initialdonor }}}$}

$V_{A}$ - volume of transport buffer in acceptor well,

Area - surface area of the insert (equals to effective growth area of the insert $-0.31 \mathrm{~cm}^{2}$ )

Time - time of the assay [drug] - concentration of test compound in acceptor well, [drug $]_{\text {initiald } d}$ - initial concentration of test compound in a donor well. $P_{a p p}$ is expressed in $10^{-6} \mathrm{~cm} / \mathrm{s}$.

The percentage recovery can be useful in interpreting the Caco-2 data. If the recovery is very low, this may indicate problems with poor solubility, binding of the compound to the test plate materials, metabolism by the Caco-2 cells, or accumulation of the compound in the cell monolayer. The percentage recovery was calculated using the following equation:

\%recovery $=\frac{C_{a c c} \times V_{a c c}+c_{d} \times V_{d}}{C_{\text {initial }, d} \times V_{d}} \times 100 C$,

$V_{\text {acc }}$ - volume of compound solution in acceptor well $\left(\mathrm{cm}^{2}\right)$

$V_{d}$-volume of compound solution in donor well $\left(\mathrm{cm}^{2}\right)$,

$C_{\text {acc }}$ - concentration of test compound in acceptor well $(\mu \mathrm{M})$,

$C_{\text {initial, }}$ - initial concentration of test compound in a donor well $(\mu \mathrm{M})$.

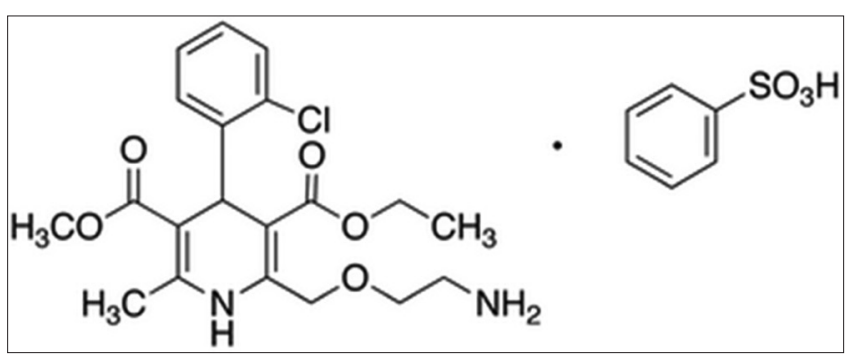

Fig. 1: The chemical structures of amlodipine besylate

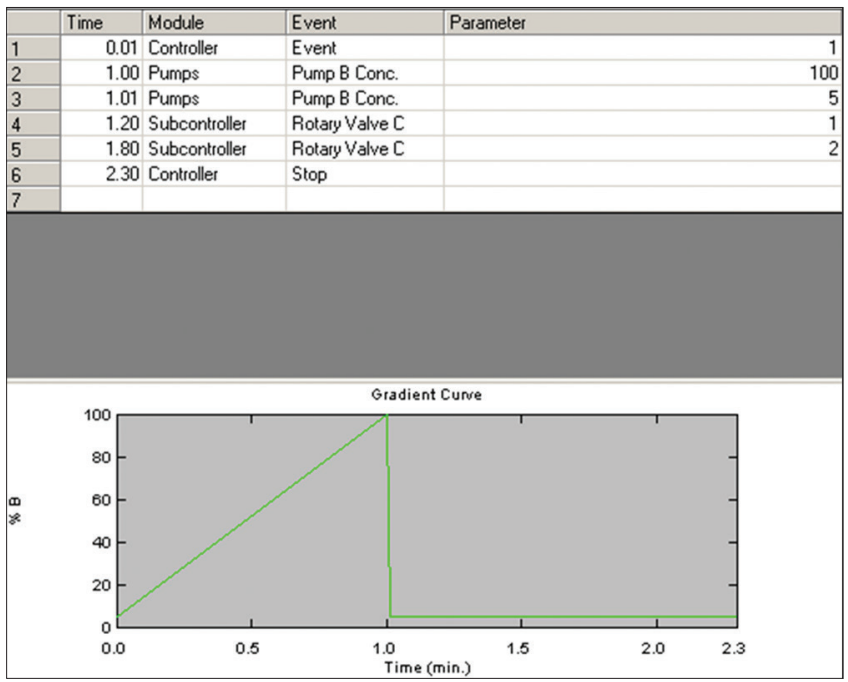

Fig. 2: Gradient curve

Table 1: Parameters of ionizer electrospray

\begin{tabular}{lll}
\hline S. No. & Parameter & Value \\
\hline 1 & Polarity & Positive \\
2 & Nebulizer gas (NEB, gas 1) & 15 \\
3 & Curtain gas (CUR) & 8 \\
4 & Collision gas (CAD) & 4 \\
5 & IonSpray voltage (IS) & 5000 \\
6 & Temperature (TEM) & 400 \\
7 & TurbolonSpray gas & 8 \\
8 & Horizontal position & 5.3 \\
9 & Lateral position & 1.3 \\
\hline
\end{tabular}


Table 2: MRM parameters

\begin{tabular}{|c|c|c|c|c|c|c|c|c|}
\hline Compound ID & Parent, m/z & Daughter, m/z & Time, ms & DP, V & FP, V & EP, V & CE, V & CXP, V \\
\hline Amlodipine & 409.315 & 238.20 & 50 & 21 & 140 & 11 & 13 & 18 \\
\hline
\end{tabular}
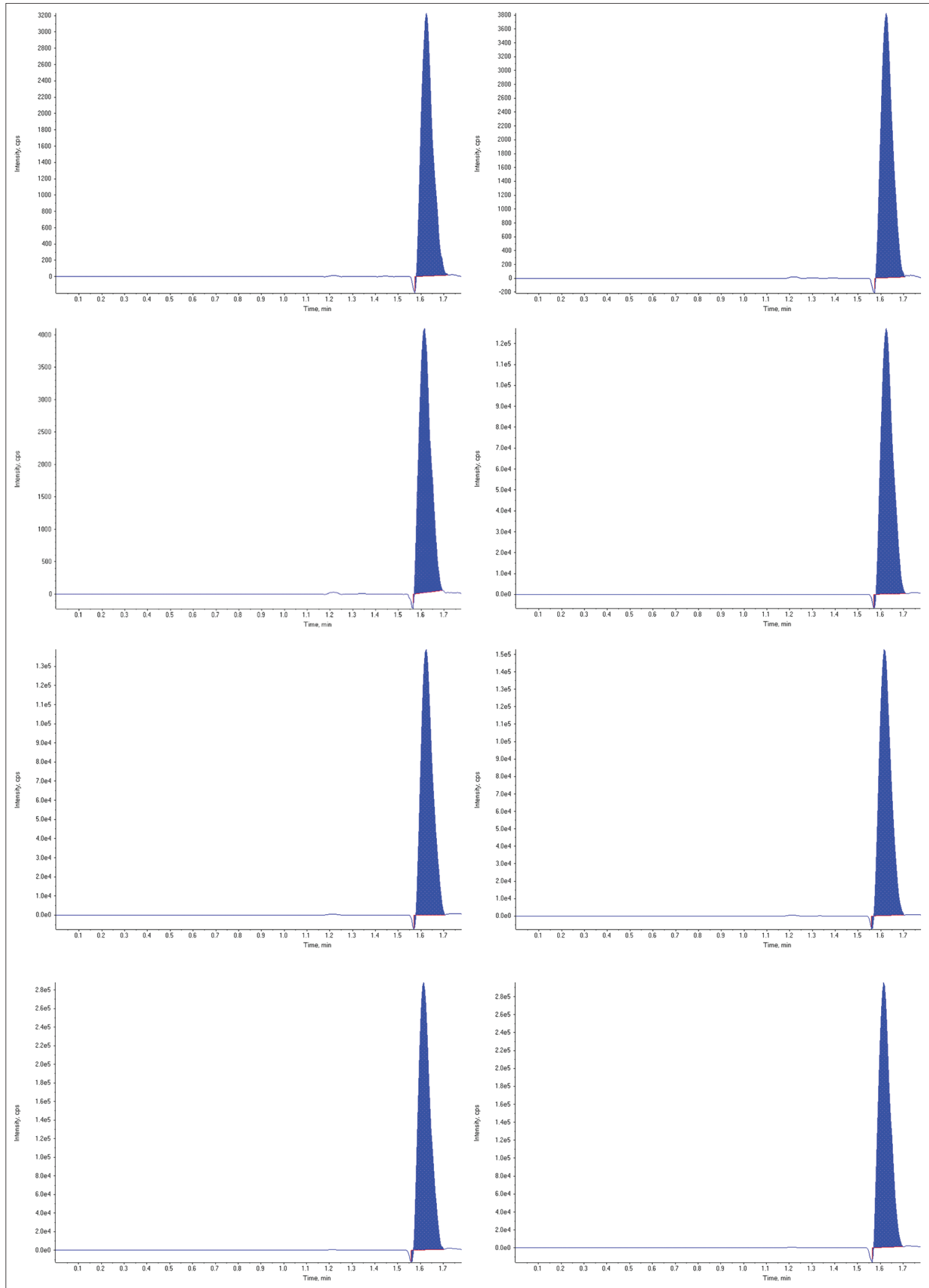

Fig. 3: Typical multiple reaction monitoring chromatograms of amlodipine 


\section{RESULTS AND DISCUSSION}

In the present study, optimization and critical evaluation of mobile phase composition (gradient), flow rate, and analytical column were important to obtain good resolution of peaks of interest from the endogenous components, which, in turn, affect reproducibility and sensitivity of the method [4-6]. The resolution of peaks was best achieved with Discovery C18, 50×2.1 mm, $5 \mu \mathrm{m}$ column. Samples were chromatographed in a gradient mode (eluent A [acetonitrilewater-formic acid, 5:95:0.1 v/v], eluent B [acetonitrile-formic acid, $100: 0.1 \mathrm{v} / \mathrm{v}])$. The initial content of the eluent $\mathrm{B}$ is $0 \%$, which increases linearly by $1.0 \mathrm{~min}$ to $100 \%$ and to $1.01 \mathrm{~min}$ returns to the initial $0 \%$. Gradient curve is shown in Fig. 2. The mobile phase was delivered at a flow rate of $0.400 \mathrm{ml} / \mathrm{min}$ into the MS ESI chamber. The injection volume was $5 \mu$ l.

Amlodipine eluted at $\sim 1.61$ min. Typical MRM chromatograms of amlodipine are shown in Fig. 3.

A-B permeability data for the test compound amlodipine besylate and three reference compounds are listed in Table 3. A-B permeability data for all the reference compounds correspond to the literature data, thus validating this study [7-9].

According to the Caco-2 test results, amlodipine appeared to have moderate to high permeability. It should be noted that the recovery value (Table 2) for amlodipine is $52.33 \%$, in contrast to the values for the control drugs. This might be due to metabolism of compound in Caco-2 cell, chemical instability, accumulation of the compound in cellular monolayer, or sorption on plastic. Therefore, the experimental Caco-2 permeability value for amlodipine should be considered as approximate. Caco-2 permeability values for amlodipine are in

Table 3: Data of A-B permeability for the test and reference compounds (at $10 \mu \mathrm{M})$

\begin{tabular}{lllll}
\hline Compound ID & \multicolumn{2}{l}{ Permeability $\left(\mathbf{1 0}^{-\mathbf{6}} \mathbf{c m} / \mathbf{s}\right)$} & \multirow{2}{*}{ Mean \pm SD $\left(\mathbf{1 0}^{-\mathbf{6}}\right)$} \\
\cline { 2 - 4 } & $\mathbf{1}$ & $\mathbf{2}$ & $\mathbf{3}$ & \\
\hline Atenolol & 1.83 & 1.99 & 1.48 & $1.77 \pm 0.26$ \\
Propranolol & 37.50 & 35.20 & 35.70 & $36.13 \pm 1.21$ \\
Quinidine & 16.50 & 23.80 & 20.00 & $20.10 \pm 3.65$ \\
Amlodipine & 6.59 & 7.8 & 8.31 & $7.57 \pm 0.88$ \\
\hline
\end{tabular}

Each value is represented as a mean \pm SD of five observations ( $n=5)$,

SD: Standard deviation, RSD: Relative standard deviation, "acceptance criteria $<2.0$

Table 4: Recovery values

\begin{tabular}{lllll}
\hline Compound ID & \multicolumn{4}{l}{ \% recovery } \\
\cline { 2 - 5 } & $\mathbf{1}$ & $\mathbf{2}$ & $\mathbf{3}$ & Mean \\
\hline Atenolol & 109.61 & 99.70 & 101.90 & 103.74 \\
Propranolol & 112.78 & 97.86 & 97.50 & 102.71 \\
Quinidine & 96.49 & 102.59 & 97.68 & 98.92 \\
Amlodipine & 48.47 & 52.07 & 56.45 & 52.33 \\
\hline
\end{tabular}

Each value is represented as a mean \pm SD of five observations $(n=5)$,

SD: Standard deviation, RSD: Relative standard deviation, \#acceptance criteria $<2.0$ agreement with BCS Class I classification for these drugs and their known high bioavailability in humans [10-13].

\section{CONCLUSION}

One of the most important challenges facing the pharmaceutical industry today is to develop high throughput, cost-effective, and predictive permeability/absorption screening models that can be used during the lead optimization process early in drug discovery. A new rapid method was developed for the determination of amlodipine from confluent Caco2 monolayers and from aqueous solution. Acquired results demonstrate that proposed strategy can be effortlessly and advantageously applied for the examination of amlodipine from Caco-2 cell monolayers.

\section{CONFLICTS OF INTEREST}

The author declared that they have no conflicts of interest.

\section{REFERENCES}

1. Balimane PV, Han YH, Chong S. Current industrial practices of assessing permeability and P-glycoprotein interaction. Bioorg Med Chem 2006;8:1-13

2. Arena A, Phillips J. Optimization of Caco-2 cell Growth and Differentiation for Drug Transport Assay Studies using a 96 Well Multiscreen Caco-2 Assay System. Millipore Protocol Note PC1060EN00P, rev. 08; 2003.

3. Srinivasan B, Kolli AR, Esch MB, Abaci HE, Shuler ML, Hickman JJ. TEER measurement techniques for in vitro barrier model systems. J Lab Autom 2015;20:107-26.

4. Logoyda L, Kondratova Y, Korobko D, Soroka Y. Development of UHPLC method for the determination of captopril in pharmaceutical dosage forms. Asian J Pharm Clin Res 2017;10:308-10.

5. Kondratova Y, Logoyda L, Voloshko Y, Abdel-Megied A, Korobko D, Soroka Y. Development and validation of HPLC-DAD method for the determination of bisoprolol in tablet dosage forms. Int J App Pharm 2017;9:54-9.

6. Logoyda L, Abdel-Megied AM, Kondratova Y, Trofimenko O, Korobko D, Dakhym I. Development and validation of HPLC method for the simultaneous determination of enalapril maleate in present of their impurities: Application to tablet analysis. Int J App Pharm 2018;10:98-102.

7. Fujikawa M, Ano R, Nakao K, Shimizu R, Akamatsu M. Relationships between structure and high-throughput screening permeability of diverse drugs with artificial membranes: Application to prediction of caco-2 cell permeability. Bioorg Med Chem 2005;13:4721-32.

8. Gertz M, Harrison A, Houston JB, Galetin A. Prediction of human intestinal first-pass metabolism of 25 CYP3A substrates from in vitro clearance and permeability data. Drug Metab Dispos 2010;38:1147-58.

9. Yazdanian M, Briggs K, Jankovsky C, Hawi A. The "high solubility" definition of the current FDA guidance on biopharmaceutical classification system may be too strict for acidic drugs. Pharm Res 2004;21:293-9.

10. Gozalbes R, Jacewicz M, Annand R, Tsaioun K, Pineda-Lucena A. QSAR-based permeability model for drug-like compounds. Bioorg Med Chem 2011;19:2615-24.

11. Hou TJ, Zhang W, Xia K, Qiao XB, Xu XJ. ADME evaluation in drug discovery. 5. Correlation of caco-2 permeation with simple molecular properties. J Chem Inform Comput Sci 2004;44:1585-600.

12. Yee S. In vitro permeability across caco-2 cells (colonic) can predict in vivo (small intestinal) absorption in man-fact or myth. Pharm Res 1997; 14:763-6.

13. Available from: http://www.cyprotex.com/admepk/in-vitro-permeability/ caco-2-permeability. 